\title{
Mobilisation of friction in unstationary flows down a model topography
}

\author{
Baptiste Blachier ${ }^{1, *}$ and Lydie Staron ${ }^{2, * *}$ \\ ${ }^{1}$ Ecole Normale Supérieure de Lyon, 69342 Lyon, France \\ ${ }^{2}$ Institut d'Alembert - CNRS - Université Paris Sorbonne, 75252 Paris, France
}

\begin{abstract}
The frictional behaviour of a series of numerical 2D granular mass flows down a model topography is analysed. Effective friction coefficients estimated from final deposits are compared with data from documented natural geophysical flows, and show a consistent behaviour as far as run-out distances are concerned. The latter is used to estimate effective friction coefficients which capture well the frictional behaviour derived from the computation of micro-mechanical stress tensors near the gravity centre. Distinguishing between the different parts of the mass while spreading, we show that the downstream part of the flow exhibits a much larger friction than the core and the tail. A dependence between friction and flow volume is however observed in each region.
\end{abstract}

\section{Introduction}

The frictional properties of model granular matter have long been perplexing experimentalists and numericians alike, even in the simplest idealised flow configurations involving the simplest idealised beads. While sustained research has finally brought some helpful light on the problem [1], the difficulties remain nearly untouched in the case of natural, namely geophysical, granular flows. The nature of the grains, their various size and shapes, the various triggering contexts, underlying processes and topography covered make the attempt at aiming at a good guess for their apparent friction properties look like a reverie, as nicely put by Haff warning us of the limitations of predictive modelling in Geomorphology [2].

Nevertheless, in this contribution, we question the frictional properties of a granular mass flowing down an idealised topography, and try to relate them to topography characteristics and final deposit geometry. Therefor, highly idealised dry grains are simulated; their behaviour is controlled only by gravity forces and contact interactions, applying a hard-sphere approximation.

We first discuss the geometrical characteristics of the deposit left by the numerical flows in relation to those left by geophysical events. Analysing the stress state in the mass while flowing, we then examine the mobilisation of macroscopic friction forces in the framework of the $\mu(I)$ theory [1]. We compare the results obtained from the computation of the stress and those estimated from the deposit left by the granular mass. Finally, we discuss the different behaviours exhibited by the various parts of the flow: head, tail and centre.

\footnotetext{
*e-mail: baptiste.blachier@ens-lyon.fr

**e-mail: lydie.staron@upmc.fr

A video is available at https://doi.org/10.48448/e1zc-v309
}

\section{Idealised numerical flows}

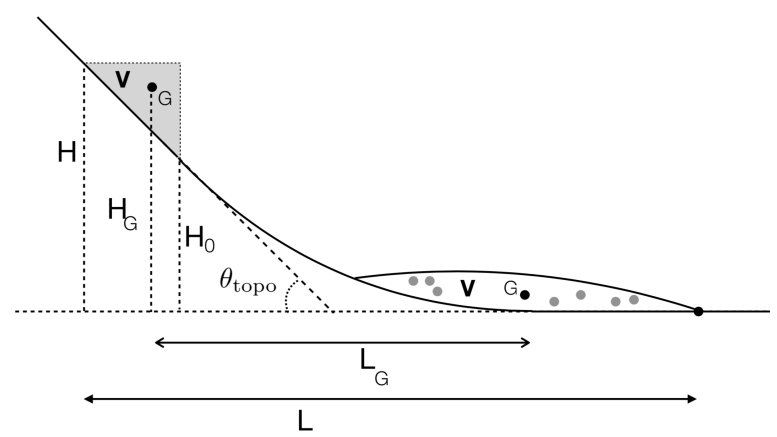

Figure 1. Granular mass and model topography over which the mass is released, left to flow and come to arrest.

The simulations were performed applying a Contact Dynamics algorithm in 2 dimensions [3]. The grains are rigid, and interact at contacts through Coulombic friction and elastic restitution. Details on the algorithm can be found in [4].

The setup consists of a compact mass of $N_{p}$ twodimensional grains forming a volume $V$, initially located on a slope of angle $\theta_{\text {topo }}$, at an initial height $H_{0}$, and allowed to flow under gravity down a simplified topography until it reaches the horizontal deposit plane and comes to arrest, as sketched in Figure 1 and illustrated in Figure 2. The curve joining the incline and the horizontal is a curvature of radius $H_{0} /\left(1-\cos \theta_{\text {topo }}\right)$. This smooth transition prevents the harsh velocity discontinuity which would happen if the flow were hitting the horizontal plane directly after falling down the incline plane [5], while the effects of centrifugal acceleration remain negligible [6]. The whole topography is made rough by gluing grains of the same size as the flowing grains. 


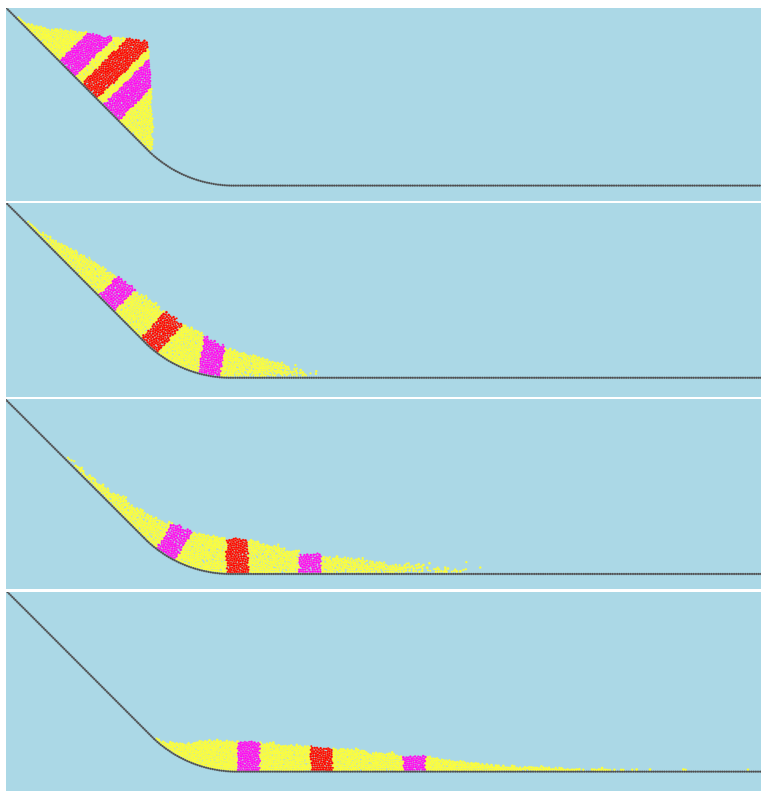

Figure 2. Successive snapshots of the flow of a granular mass $\left(N_{p}=1452\right)$. In red and pink are shown the neighbourhood of the main, and upstream and downstream secondary centres of gravity $G, G^{\prime}$ and $G^{\prime \prime}$, over which mean quantities are evaluated.

Table 1. Simulations performed

\begin{tabular}{lclc}
\hline Volume (in nb. grains) & $H_{0}$ & $\theta_{\text {topo }}$ & $\mathrm{Nr}$ of runs \\
\hline$[655: 7882]$ & $0.08 \mathrm{~m}$ & $45 \mathrm{deg}$ & 31
\end{tabular}

We systematically vary the initial volume $\mathrm{V}$ of the flowing mass by varying the number of grains, from 655 to 7882 grains; its initial height is set to $H_{0}=0.08 \mathrm{~m}$, initial slope angle to $\theta_{\text {topo }}=45 \mathrm{deg}$, and are not varied. After the mass has flown and spread, we measure the horizontal distance between the top of the initial position of the mass and the final position of the front, denoted $L$ in the following, as an analogue of the runout distance measured in situ for geophysical events between the top of the break-away scar and the distal end of of the runout deposit [5].

Grains are disks with a mean diameter $d=0.005 \pm 0.001 \mathrm{~m}$; they interact through simple Coulombic friction with a coefficient $\mu_{\text {micro }}=0.3$, and a coefficient of restitution at collisions $e=0.1$; gravity was set to $g=9.8 \mathrm{~m} \mathrm{~s}^{-2}$.

The set of simulations performed and analysed in the following is presented in table 1 .

\section{3 "Geometrical" effective friction}

To compensate for the impossibility to measure directly the dissipative properties of any given natural flows, geomorphologists estimate the mobility of the mass based on the (often only) knowledge available for the event, namely the deposit left by the flow. A "travel angle", giving an effective friction characterising the motion if seen as a "solid" one, can be derived from the runout distance $L$ and the maximum initial height of the tail of the flow: $\mu_{e f f}=H / L[7,8]$. The inverse defines the a)

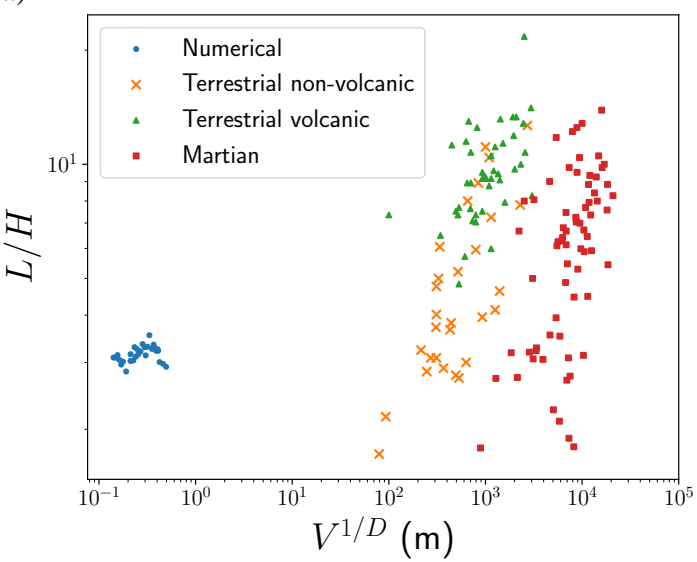

b)

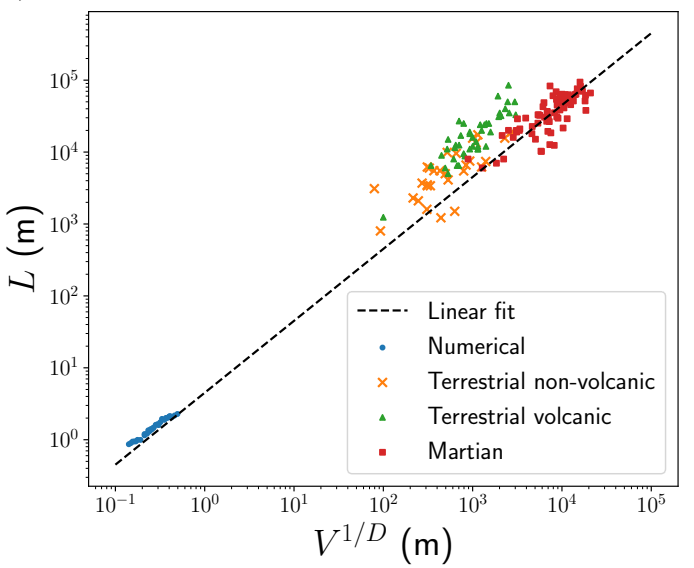

Figure 3. Data from geophysical events from Legros 2002 [9], and data points from the simulations from table 1, showing (a) the effective mobility $\mathcal{M}=1 / \mu_{\text {eff }}=L / H$ and (b) the runout distance $L$ as a function of the volume $V^{1 / D}$, where $D=2$ for numerics and $D=3$ for real events.

mobility $\mathcal{M}=1 / \mu_{\text {eff }}$.

Figure 3 shows data from real events (compiled in Legros 2002 [9]) together with the results of our simulations: the mobility $\mathcal{M}$ is plotted as a function of the volume $V$ of the event/simulation, with $V$ held to the power $1 / D$ with $D=2$ for 2D-numerics and $D=3$ for $3 \mathrm{D}$-geophysical cases so that data can be compared consistently. We observe three distinct clouds of points spanning a large interval, showing however that mobility tends to increase (namely diminishing friction) with increasing volume, but allowing no further insight. Yet, plotting the runout distance $L$ alone as a function of $V^{1 / D}$ shows a well defined trend suggesting that idealised 2D numerics and $3 \mathrm{D}$ real cases, in spite of the variety of underlying physics and difference of scale, show a common trend where geometrical spreading is concerned, as suggested by Davies (1982) [10], and later verified for numerics in [11]. We now turn towards simulations to analyse the frictional behaviour of the granular mass in terms of stress state, and compare it with the geometrical estimation given by the mobility $\mu_{\text {eff }}=1 / \mathcal{M}$. 


\section{Friction mobilisation}

\subsection{Obeying the $\mu(I)$ law?}

To explore the mobilisation of friction in the bulk of the granular mass while flowing, we focus on a region around the gravity centre $G$ and compute the stress tensor in this area (Figure 2). Therefor, we consider a slice normal to the topography, of width $W=10 d$ and volume $\mathcal{V}$, centred on $G$. We compute over all contacts $c$ and grains $n$ situated in $\mathcal{V}$ :

$$
\sigma=\frac{1}{\mathcal{V}} \sum_{c \in \mathcal{V}} \vec{f}^{c} \otimes \vec{l}^{c}
$$

where $\vec{f}$ is the contact force and $\vec{l}$ is the contact distance defined as the distance between the two grains centres. We are thus able to form the friction coefficient $\mu=\tau / P$ for the volume $\mathcal{V}$, where $\tau$ is the shear stress and $P$ the pressure. The contribution of the kinetic stress tensor is neglected here, based on earlier computation of the contribution of dynamic stress in chute flow showing small comparative values [12].

Knowing the mean velocity $\langle u\rangle$ of the slice and its height $h$ at each moment of the simulations, we are able to evaluate the inertial number $I=d\langle u\rangle / h \sqrt{P / \rho}$ ( $P$ being the pressure and $\rho$ the density) for each run. We thus plot the friction coefficient $\mu$ against $I$ averaged over successive time intervals of $\Delta t=0.125$ s corresponding only to dynamical states (namely filtering out onset and arrest). Bringing together all simulations points from all simulations in Figure 4, we observe a cloud of points roughly resembling the familiar trend [1]. The best fit for $\mu=\mu_{1}+\left(\mu_{2}-\mu_{1}\right) /\left(\frac{I_{0}}{I}+1\right)$ gives a tiny static friction $\mu_{1}=0.05$ and a dynamical friction $\mu_{2}=0.33$. However, a fit with $\mu_{1}=0.19$ and $\mu_{2}=0.36$ is acceptable, and coincides with a larger value of $\mu_{1}$ more commonly observed for dry granular matter [1]. We hence conclude that, despite their unstationary and non-uniform nature, the granular avalanches simulated here fall in the framework of the $\mu(I)$ dependence. Nevertheless, addressing the contribution of the kinetic stress tensor would be of interest.

\subsection{Head or tail?}

The coefficients of friction computed so far involve only the contacts in the neighbourhood of the gravity centre $G$. It thus does not seem unreasonable to compare them with an effective friction defined geometrically from the initial and final positions of $G$. In the same way that we have defined $\mu_{e f f}=H / L$, we define $\mu_{e f f}^{G}=H_{G} / L_{G}$ for each of the $n$ simulations performed. Since the runout $L$ is much larger than the position $L_{G}$ by definition, while initial heights $H$ and $H_{G}$ are somewhat comparable, we expect the effective friction $\mu_{e f f}^{G}$ to be much larger than $\mu_{e f f}$; this is indeed observed in Figure 5 where both are plotted as a function of the volume of the flow.

To compare the coefficient of friction $\mu$ computed from the stress state in the neighbourhood of the gravity center $G$ with the effective frictions $\mu_{e f f}^{G}$ and $\mu_{e f f}$, we select for each simulation a time window where the flow is actually

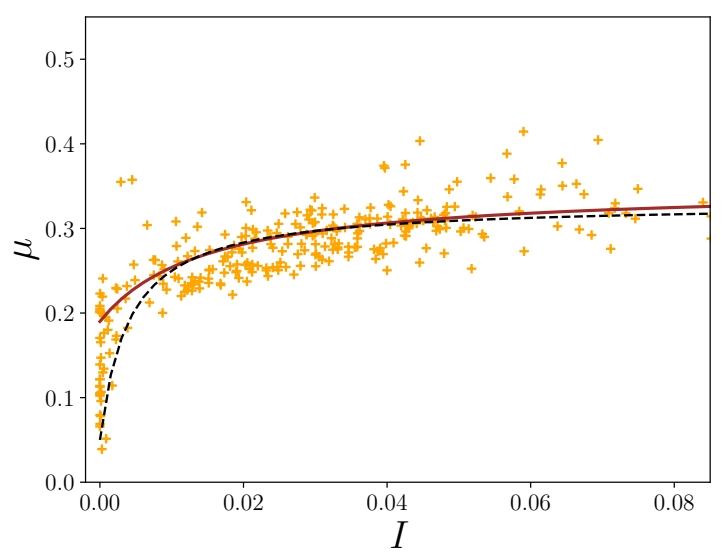

Figure 4. Coefficient of friction $\mu$ computed in the neighbourhood of the centre of mass plotted against the inertial number $I$. The best fit for $\mu=\mu_{1}+\left(\mu_{2}-\mu_{1}\right) /\left(\frac{I_{0}}{I}+1\right)$ is shown in dotted line, and an acceptable trend is obtained in red for more classical values for the static friction $\mu_{1}$ (see text).

dynamical, excluding onset and arrest phases, and apply expression (1). We then plot all points $\mu, \mu_{\text {eff }}$ and $\mu_{\text {eff }}^{G}$ as a fonction of the flow volume $V$ in Figure 5. Unexpectedly, $\mu$ does not at all compare with $\mu_{e f f}^{G}$. By contrast, it coincides with the effective friction $\mu_{\text {eff }}$ derived from the runout distance.

If the values of $\mu$ computed here were relevant for the whole flow (and not only for the area around the gravity centre), that would mean that the geometry of the final deposit is a straightforward translation of the dissipation occurring in the flow. To discuss this point, we examine the friction mobilisation in the various regions of the flowing mass: centre, head and tail.

\subsection{Two regimes showing up}

The highly unstationary nature of the flow, with a mass strongly deforming while flowing, and a topography evolving from incline to horizontal, means that dissipative processes may depend on which part is considered, ranging from a slow bulk motion upstream to a rapid thin shear flow downstream. To clarify this issue, we consider now two secondary gravity centres, upstream tail $G^{\prime}$ and downstream front $G^{\prime \prime}$. We compute the corresponding coefficients of friction $\mu^{\prime}$ and $\mu^{\prime \prime}$ from the stress state in local neighbourhoods (see Figure 2) in the same way that we have computed $\mu$ in the neighbourhood of $G$ in subsection 4.2. Their values is displayed in Figure 6 as a function of the volume $V$ involved in the flow.

Two aspects can be noticed. First, the upstream tail $G^{\prime}$ shows the same feature as the main gravity centre $G$, while downstream front $G^{\prime \prime}$ exhibits much larger values of friction. This suggests that the tail of the flow follows the behaviour of the middle core, while the front has an independent, significantly different, behaviour of its own. This also implies that mean values are dominated by what happens in the upstream part of the flow, and say little on the processes occurring downstream. This 


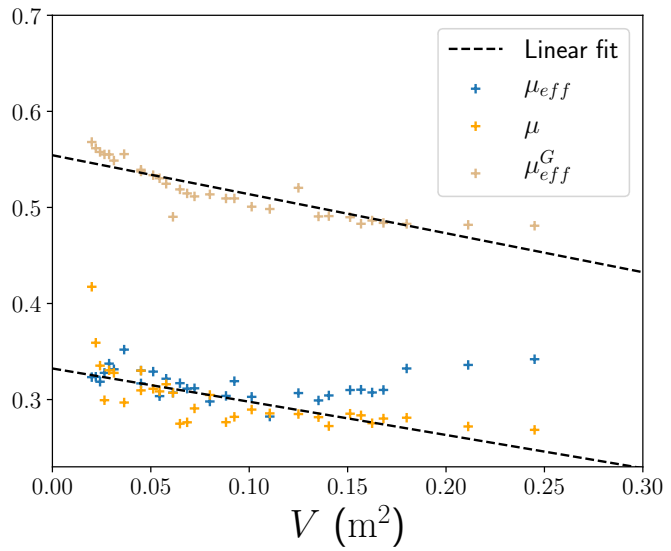

Figure 5. Effective coefficient of friction derived from the final runout $\mu_{e f f}=H / L$ and from the final position of the centre of gravity $\mu_{e f f}^{G}=H_{G} / L_{G}$, and coefficient of friction $\mu=\tau / P$ computed from the stress tensor in the neighbourhood of the gravity centres.

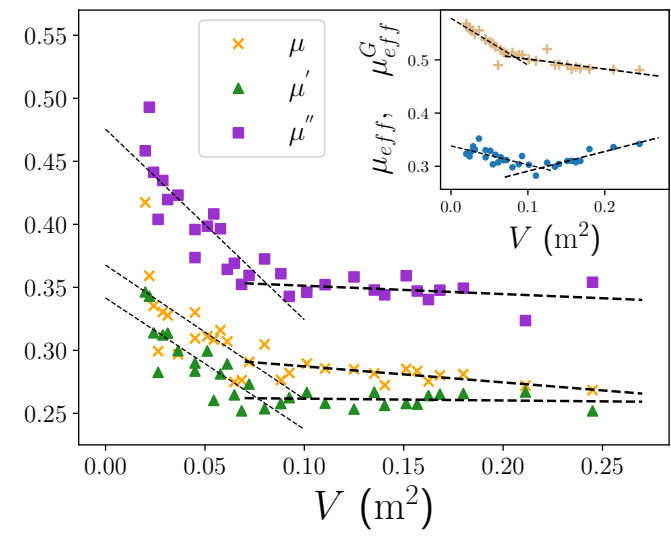

Figure 6. Coefficient of friction computed from the stress tensors in the neighbourhood of the gravity centre $G$ and the secondary upstream and downstream ones $G^{\prime}$ and $G^{\prime \prime}$ as a function of the volume $V$ involved during the flow.

coincides with a bouncy dynamics at the front, efficiently dissipating energy through a collisional regime, while the tail, moving in a denser regime, ressembles more a smooth bulk translation.

Second, for all three gravity centres, two regimes seem to emerge depending on the volume involved. For smaller volumes (here $V \lesssim 0.09 \mathrm{~m}^{2}$ ), friction coefficients decrease distinctly with $V$, but reach a plateau afterward. This suggests that large volumes no longer facilitate motion after a threshold is reached. Note that this departs from the established - yet still discussed and explored - volumeinduced lubrication theory/observation in Geosciences. This point needs systematic verification while discussing not only the volume of the flow, but the geometry of the topography, in the spirit of [11]. Increasing volume favours motion through spreading over sliding, namely bulk volume dissipative processes over bottom boundary interactions. How this may determine the macroscopic behaviour, and how this compete with other factors (roughness, rheology, grain-size distribution... ) remains an open question.

\section{Conclusion}

In this contribution, we have analysed the frictional behaviour of a series of numerical 2D granular mass flows down a model topography. Following Geoscience practice, we have estimated effective friction coefficients from the geometry of final deposits, and compare it with data from natural geophysical flows from the literature. $\mathrm{Nu}-$ merical and natural data show a consistent behaviour as far as run-out distance is concerned.

We then analyse the mobilisation of friction from the computation of micro-mechanical stress tensors, namely the ratio of the deviatoric part to the pressure, and disclose a behaviour consistent with the $\mu(I)$ dependence. We observe that the friction computed near the gravity centre is well estimated from analysing the deposit geometry in terms of the run-out distance. However, defining upstream and downstream secondary gravity centres, the computation of stress tensors in the vicinity of each reveals that the downstream part of the flow actually exhibits a much larger friction coefficient, presumably due to a collisional flow regime. All three gravity centres, however, show a similar dependence on the flow volume, departing from the volume-induced lubrication theory. Further simulations, varying the topography characteristics, are needed to clarify this issue.

\section{References}

[1] GDR MiDi, Eur. Phys. J. E 14, 341-365 (2004)

[2] P. K. Haff, The scientific nature of Geomorphology: Proceedings of the 27th Binghamton Symposium in Geomorphology (1996)

[3] J.-J. Moreau, European Journal of Mechanics, A/Solids 13, 93-114 (1994)

[4] F. Radjai, V. Richefeu, Mechanics of Materials 41, 715-728 (2009)

[5] T.R. Davies, M.J. McSaveney, Can. Geotech. J. 36, 313-320 (1999)

[6] L. Staron, Geophys. J. Int 172, 455-463 (2007)

[7] D. M. Cruden, D.J. Varnes, Transportation research board special report 247, 36-75 (1996)

[8] A. Heim, Zurich Vierteljarhrsschrift 77, 218 (1932)

[9] F. Legros, Engineering Geology 63, 301-331 (2002)

[10] T. R. Davies, Rock Mech. 15, 9-24 (1982)

[11] L. Staron, E. Lajeunesse, Geophys. Res. Lett. 36, L12402 (2009)

[12] L. Staron, J. C. Phillips, Stress partition and microstructure in size-segregating granular flows, Phys. Rev. E 92, 022210 (2015) 